\title{
A promising strategy for overcoming MDR in tumor by magnetic iron oxide nanoparticles co-loaded with daunorubicin and 5-bromotetrandrin
}

\author{
This article was published in the following Dove Press journal: \\ International Journal of Nanomedicine \\ 26 September 2011 \\ Number of times this article has been viewed
}

\author{
Jian Cheng ${ }^{*}$ \\ Jun Wang ${ }^{2 *}$ \\ Baoan Chen' \\ Guohua Xia' \\ Xiaohui Cai' \\ Ran Liu' \\ Yanyan Ren' \\ Wen Bao' \\ Xuemei Wang ${ }^{3}$ \\ 'Department of Hematology, \\ Zhongda Hospital, Medical School, \\ Southeast University, Nanjing, People's \\ Republic of China; ${ }^{2}$ Department \\ of Hematology and Oncology, \\ Nanjing Children's Hospital, Nanjing \\ Medical University, Nanjing, People's \\ Republic of China; ${ }^{3}$ National Key \\ Laboratory of Bioelectronics \\ (Chien-Shiung Wu Laboratory), \\ Southeast University, Nanjing, \\ People's Republic of China \\ *These authors have contributed \\ equally to this work
}

\begin{abstract}
To overcome both the dose-limiting side effects of conventional chemotherapeutic agents and the therapeutic failure resulting from multidrug resistance (MDR) and minimize adverse effects of chemotherapy agents, a novel chemotherapy formulation of magnetic nanoparticles co-loaded with daunorubicin and 5-bromotetrandrin (DNR/BrTet-MNPs) was developed, and its effect on MDR leukemic cells was explored. After the DNR and $\mathrm{Br}$ were co-loaded onto a pluronic-stabilized and oleic acid-modified magnetic nanosystem, the physical characteristic and drug-loading capacity were evaluated. The cell toxicity of the self-prepared DNR/BrTet-MNPs formulation was then determined by MTT (3-[4,5-dimethylthiazol-2-yl]-2,5-diphenyltetrazolium bromide) assay; the cellular uptake of drug was demonstrated by fluorescent microscope. Lastly, the transcription of $\mathrm{mdr} 1$ and the expression of P-glycoprotein (P-gp) were detected by the reverse transcription reaction and western blotting assay, respectively. The results showed that the selfprepared DNR/BrTet-MNPs formulation possessed a sustained release of drug and displayed a dose-dependent antiproliferative activity on MDR leukemia K562/A02 cells. It also enhanced the accumulation of intracellular DNR in K562/A02 cells and downregulated the transcription of the mdr1 gene and the expression of P-gp. These findings suggest that the remarkable effect of the novel DNR/BrTet-MNPs formulation, acting as a drug depot system for the sustained release of the loaded DNR and BrTet, on multidrug resistance leukemia K562/A02 cells would be a promising strategy for overcoming MDR.
\end{abstract}

Keywords: multidrug resistance, targeting drug delivery system, leukemia

\section{Introduction}

Owing to multidrug resistance (MDR), systematic chemotherapy is limited in leukemia treatment. ${ }^{1}$ Recent exciting data suggest that the overexpression of adenosine triphosphate-dependent drug efflux pump on the surface of tumor cells, P-glycoprotein (P-gp/ABCB1), is the main cause of MDR. ${ }^{2} \mathrm{P}$-gp, coded by the mdr1 gene, effluxes drug and decreases intracellular drug dose, which reduces cytotoxicity of antitumor drugs and thus induces MDR. ${ }^{3}$ Therefore, exploiting P-gp inhibitors has the potential of overcoming MDR and enhancing chemotherapy efficacy. ${ }^{4}$ Unfortunately, these compounds are often nonspecific and have low efficiency and/or high toxicity; as such, Phase 3 clinical trials of these agents are largely disappointing. ${ }^{5-9}$ Therefore, searching for more efficient and lower toxic MDR reversal agents is our main purpose in refractory tumor therapy.

Tetrandrine (Tet), a bis-benzylisoquinoline alkaloid, is the extract component from the root of Stephania tetrandra S. Moore (or Fenfangji) in the Menispermaceae family
Correspondence: Baoan Chen Department of Hematology, Zhongda Hospital, Medical School, Southeast University, Nanjing 210009,

People's Republic of China

Tel +862583272006

Fax +86 2583272011

Email cba8888@hotmail 
of traditional Chinese herbs. ${ }^{10}$ A number of accumulated evidences indicate that Tet has been shown to be a $\mathrm{Ca}^{2+}$ channel antagonist, and it has reversal effect on P-gp-mediated MDR. ${ }^{11,12}$ Moreover, 5-bromotetrandrine (BrTet) is a bromized derivative of Tet, which is more efficient than Tet at modulating MDR in vitro. ${ }^{13}$ Therefore, co-administration of conventional chemotherapy with an MDR reversal agent enhances their antitumor efficacy and reduces their side effects. However, it is necessary to minimize the exposure of normal cells to inhibitor and anticancer drugs, and to co-localize both of them in the tumor cells. Currently, nanotechnology has shown tremendous progress in target-specific delivery of drugs in the body. ${ }^{14}$ The targeting drug-delivery system is far more effective than conventional chemotherapy, benefiting from decreasing toxicity of drugs to normal tissues, prolonging circulation half-life in plasma, enhancing drug stability, improving aqueous solubility, and increasing tumor site selectivity. ${ }^{15,16}$ Due to their biocompatibility and biodegradability, superparamagnetic iron oxide nanoparticles have been widely used in various biomedical applications, ${ }^{17}$ such as a carrier for anticancer drugs. Daunorubicin (DNR) is one of the anthracyclines and a substrate for P-gp, ${ }^{18}$ which is the essential part of first-line chemotherapy in the treatment of many solid and hematological malignancies, ${ }^{19}$ but the clinical use of DNR has been impeded by its serious side effects, such as cardiotoxicity and drug resistance. ${ }^{20}$ To overcome both the dose-limiting side effects of conventional chemotherapy and the therapeutic failure of MDR, a novel drug-delivery formulation, magnetic iron oxide nanoparticles co-loaded with DNR and BrTet (DNR/BrTet-MNPs) was developed, and its reversal efficacy on K562/A02 cells was evaluated in vitro.

\section{Materials and methods Main drugs and chemicals}

The following materials were purchased: DNR hydrochloride (DNR $\cdot \mathrm{HCl}$ ) (Huifengda Chemical Co, Ltd, Jinan, China); BrTet (Kanghong Phamaceuticals Inc, Chengdu, China); doxorubicin (Hisun Phamaceutical Co, Zhejiang, China); iron chloride hexagydrate $\left(\mathrm{FeCl}_{3} \cdot 6 \mathrm{H}_{2} \mathrm{O}\right)$, iron sulfate heptahydrate $\left(\mathrm{FeSO}_{4} \cdot 7 \mathrm{H}_{2} \mathrm{O}\right)$, ammonium hydroxide $\left(\mathrm{NH}_{3} \cdot \mathrm{H}_{2} \mathrm{O}\right)$, oleic acid (OA), and absolute ethanol (Sinopharm Chemical Reagent Co, Ltd, Shanghai, China); Pluronic ${ }^{\circledR}$ F-127, 3-(4, 5-dimethylthiazol2-yl)-2, 5-diphenyltetrazolium bromide (MTT) (Sigma Aldrich, St Louis, MO); 4', 6-diamidino-2-phenylindole (DAPI) (Beyotime Institute of Biotechnology, Shanghai, China); randomly primed polymerase chain reaction (RP-PCR) kit (Takara Biotechnology, Dalian, China); mouse monoclonal anti-human
P-gp and glyceraldehyde 3-phosphate dehydrogenase (GAPDH) (Santa Cruz Biotechnology, Santa Cruz, CA).

\section{Synthesis of DNR/BrTet-MNPs}

According to the authors' previously described method, ${ }^{21}$ OA-coated and pluronic-stabilized iron oxide nanoparticles were synthesized, both hydrophobic DNR and hydrophobic BrTet were prepared by adding triethylamine into DNR or BrTet hydrochloride methanolic solution, and a combination of $\mathrm{DNR} / \mathrm{BrTet}(\mathrm{w} / \mathrm{w}=1: 1)$ was added dropwise into the above mentioned aqueous dispersion of MNPs, which was being stirred vigorously on a magnetic stirring plate overnight to make the drug participate into OA layer. ${ }^{22}$ Finally, the product (DNR/BrTet-MNPs) was washed twice with deionized water to remove the drug adsorbed on the surface of the MNPs. A magnet was put outside of the vial for 5 hours, which attracted the drug-loaded MNPs, and then the liquid was removed carefully. The drug-loaded MNPs were then resuspended in deionized water and washed twice to obtain the synthesized DNR/BrTet-MNPs.

\section{Physical characteristic measurements}

The sample was suspended in water and dropped on a carbon-coated copper grid to analyze the morphology of OA-coated and pluronic-stabilized iron oxide nanoparticles under transmission electron microscope (JEM-2100; JEOL, Tokyo, Japan). Meanwhile, the hydrodynamic diameter and zeta potential of DNR/BrTet-MNPs were determined by dynamic light scattering (ZS90; Malvern Instruments, Worcestershire, UK) and zeta plus potential analyzer (Brookhaven Instruments, Holtsville, NY), respectively. Furthermore, the magnetic properties of drug-loaded MNPs were measured using a vibration sample magnetometer (VSM) integrated in a physical property measurement system (PPMS-9; Quantum Design, San Diego, CA) up to 50,000 Oe at $300 \mathrm{~K}$.

\section{Studying drug loading and release}

To determine DNR loading, less than $2 \mathrm{mg}$ lyophilized drugloaded MNPs were dispersed in $2 \mathrm{~mL}$ methanolic solution $(12.5 \%)$ in chloroform $(\mathrm{v} / \mathrm{v})$. The solution was shaken for 24 hours and then centrifuged at $16,000 \mathrm{rpm}$ for 10 minutes at $4{ }^{\circ} \mathrm{C}$. Volumes of $100 \mu \mathrm{L}$ supernatant were diluted to $2 \mathrm{~mL}$ methanolic-chloroform solution and analyzed using a Shimadzu HPLC system (Shimadzu, Kyoto, Japan) at detection/excitation wavelengths of $480 / 560 \mathrm{~nm}$. To determine BrTet loading, the lyophilized drug-loaded MNPs were dispersed in $2 \mathrm{~mL}$ methanolic solution and shaken as above; 
$1 \mathrm{~mL}$ supernatant was diluted to $4 \mathrm{~mL}$ methanolic solution and measured at wavelength of $281 \mathrm{~nm}$ using an ultraviolet spectrophotometer (UV-3600; Shimadzu). The DNR or BrTet loading capacity (LC) and encapsulation efficiency (EE) of the nanoparticles were calculated as:

and

$$
\mathrm{LC}=W_{e} / W_{p} \times 100 \%
$$

$$
\mathrm{EE}=W_{e} / W_{t} \times 100 \%
$$

where $W_{e}$ is the amount of DNR or BrTet encapsulated in nanoparticles, $W_{p}$ is the weight of drug-loading MNPs, and $W_{t}$ is the total amount of DNR or BrTet added.

For the in vitro drug release study, a certain amount of lyophilized drug loaded-MNPs were dispersed in $2.5 \mathrm{~mL}$ Tween ${ }^{\circledR}$-PBS (phosphate buffered saline) $(\mathrm{pH}=7.4$, containing $0.1 \% \mathrm{w} / \mathrm{v}$ Tween-80), and then placed in a dialysis membrane bag with a molecular weight cutoff of 8000-14,000 $\mathrm{Da}$. The bag was bathed in a tube with $2.5 \mathrm{~mL}$ Tween-PBS solution, and the tube was incubated in a shaking water bath at $37^{\circ} \mathrm{C}$. The buffer was withdrawn at different time intervals and complemented with the fresh buffer. The amount of DNR and that of BrTet in buffer were analyzed as above.

\section{Cell culture}

Human chronic myeloid leukemia resistant cell line to doxorubicin, K562/A02, was received as a gift from the Institute of Hematology, Chinese Academy of Medical Sciences. Cells were cultured in RPMI (Roswell Park Memorial Institute) 1640 medium containing 10\% heat-inactivated newborn bovine serum, 10 mM HEPES (4-[2-hydroxyethyl]1-piperazineethanesulfonic acid), $100 \mathrm{U} / \mathrm{mL}$ penicillin, and $100 \mu \mathrm{g} / \mathrm{mL}$ streptomycin at $37^{\circ} \mathrm{C}$ in a humidified $5 \% \mathrm{CO}_{2}$ incubator. For maintaining MDR phenotype, K562/A02 was cultured in the medium containing $1 \mu \mathrm{g} / \mathrm{mL}$ doxorubicin at least 1 week before use.

\section{Cytotoxicity assay}

K562/A02 cells were plated at a density of 20,000 cells per well in 96-well plates. Different concentrations of DNR alone or its combination with MNPs $(0.1-50.0 \mu \mathrm{g} / \mathrm{mL})$, and coadministration of DNR and BrTet (DNR:BrTet $=1: 1 \mathrm{w} / \mathrm{w}$ ) or MNPs $(0.05-20.00 \mu \mathrm{g} / \mathrm{mL})$ were added. After treatment for 48 hours, $20 \mu \mathrm{L}$ MTT solution $(5 \mathrm{mg} / \mathrm{mL})$ was added to each well and incubated for another 4 hours at $37^{\circ} \mathrm{C}$, the formazan salts were dissolved with $150 \mu \mathrm{L}$ dimethyl sulfoxide, and the absorbance at $450 \mathrm{~nm}$ was measured using a microplate reader
(Multiskan MK3; Thermo Scientific, Boston, MA). The cell viability was calculated as $\left(\mathrm{OD}_{\text {treated group }} / \mathrm{OD}_{\text {control group }}\right) \times 100 \%$, and half-maximal inhibitory concentration $\left(\mathrm{IC}_{50}\right)$ values were calculated.

\section{Fluorescence microscope}

K562/A02 cells were collected and smeared after being cultured as above. The cells were fixed with methanol for 15 minutes and then stained with fluorochrome dye DAPI. Morphologic characteristics were observed under a fluorescence microscope (X51; Olympus, Tokyo, Japan), with a peak excitation wavelength of $340 \mathrm{~nm}$.

\section{Intracellular DNR accumulation with rhodamine 123 staining}

Rhodamine 123 (R123), cellular accumulation of P-gp substrate, was examined to evaluate the function of P-gp of K562/A02 cells. In brief, after being treated as above, K562/A02 cells were washed with ice-cold PBS and then incubated with $100 \mu \mathrm{g} / \mathrm{L}$ R123 for 60 minutes. After being washed and suspended in $500 \mu \mathrm{L}$ PBS, each sample was determined using a flow cytometer at excitation/emission wavelengths of $488 / 575 \mathrm{~nm}$. The relative R123 fluorescence intensity (FI) was calculated as $\mathrm{FI}_{\text {treated group }} / \mathrm{FI}_{\text {control group }}$. Those cells treated with RPMI 1640 alone were used as negative control.

\section{RT-PCR analysis of mdr I mRNA}

The K562/A02 cells were incubated as above. Total RNA extraction was conducted with TRIzol ${ }^{\circledR}$ (Invitrogen, Carlsbad, CA), and RT-PCR was performed according to manufacturer's instructions. The reverse transcription reactions were performed using TaKaRa RNA PCR Kit as previously described. ${ }^{23}$ The designed PCR primers included the mdrl primers (forwards 5'-TGGTTTGATGTGCACGATGTTGGG-3', reverse 5'-AGATCAGCAG GAAAGCAGCACCTA-3') and the GAPDH primers (forwards 5'-GCCACATCGC TCAGACAC-3', reverse 5'-CATCACGCCACAGTTTCC-3'). The amplified PCR products were $436 \mathrm{bp}$ for mdr1 and $614 \mathrm{bp}$ for GAPDH. The newly synthesized cDNA was amplified by PCR, and the conditions were $94^{\circ} \mathrm{C}$ for 30 seconds, $56^{\circ} \mathrm{C}$ for 30 seconds, and $72^{\circ} \mathrm{C}$ for 1 minute. There was pre-denaturing at $94^{\circ} \mathrm{C}$ for 2 minutes and final extension at $72^{\circ} \mathrm{C}$ for 7 minutes. The obtained PCR products were visualized on $1.5 \%$ agarose gels with ethidium bromide staining, and densitometry analysis was performed using ScnImage software (Scion Corporation, Frederick, MD). 


\section{Western blotting assay}

K562/A02 cells were incubated as above. The harvested cells were lysed in lysis buffer containing $25 \mathrm{mM}$ Tris- $\mathrm{HCl}$ $(\mathrm{pH}=7.4), 150 \mathrm{mM} \mathrm{NaCl}, 1 \%$ Triton $^{\circledR} \mathrm{X}-100,5 \mathrm{mM}$ EDTA (ethylenediaminetetraacetic acid), $5 \mathrm{mM}$ EGTA (ethylene glycol tetraacetic acid), $10 \mathrm{mM} \mathrm{NaF}, 1 \mathrm{mM}$ phenylmethanesulfonylfluoride, $0.5 \% \mathrm{NP}-40,10 \mu \mathrm{g} / \mathrm{mL}$ aprotinin, $10 \mu \mathrm{g} / \mathrm{mL}$ leupeptin, and $1 \mathrm{mM}$ pepstatin for 15 minutes at $4^{\circ} \mathrm{C}$, then centrifuged at 10,000 rpm for 5 minutes. The supernatant was collected and the amount of protein was measured using Bio-Rad protein assay (Bio-Rad, Hercules, CA). Samples containing $25 \mu \mathrm{g}$ protein were separated by $10 \%$ SDS-PAGE (sodium dodecyl sulfate polyacrylamide gel electrophoresis) and then electro-transferred onto nitrocellulose filter. Five percent nonfat milk was used to block the nonspecific binding sites for 1 hour. Mouse monoclonal anti-human P-gp (1:200) or GAPDH (1:400) antibodies were incubated to the membrane overnight at $4{ }^{\circ} \mathrm{C}$, followed by horseradish peroxidase-labeled rabbit-mouse immunoglobulin $\mathrm{G}$ (1:5000) as a secondary antibody. The blots were calculated by densitometry scans (ECL system, Amersham, UK) according to manufacturer's instructions.

\section{Statistical analysis}

All the data were expressed as mean \pm standard deviation, and analyzed using SPSS software (v 13.0; SPSS Inc, Chicago, IL). Differences among various groups were evaluated using one-way analysis of variance. All the reported $P$ values were two-tailed, and those $<0.05$ were considered to be statistically significant.

\section{Results}

\section{Physical characteristics of drug-loaded magnetic nanoparticles}

The range of magnetic iron oxide nanoparticles was 10-20 nm, and the clusters of nanoparticles resulting from dehydration were also observed in local fields under transmission electron microscopy. The lattice structure of iron oxide nanoparticles under high-resolution electron microscope demonstrated that the nanoparticles were composed of magnetite $\left(\mathrm{Fe}_{3} \mathrm{O}_{4}\right)$ (Figure 1A). The synthesized DNR/BrTet-MNPs yielded a hydrodynamic average size of $135 \mathrm{~nm}$ with polydispersity index of 0.185 by dynamic light scattering (Figure 1B). The zeta potential of DNR/BrTet-MNPs in water was $-27.02 \pm 0.75 \mathrm{mV}$, and the drug-loaded MNPs were superparamagnetic with a saturation
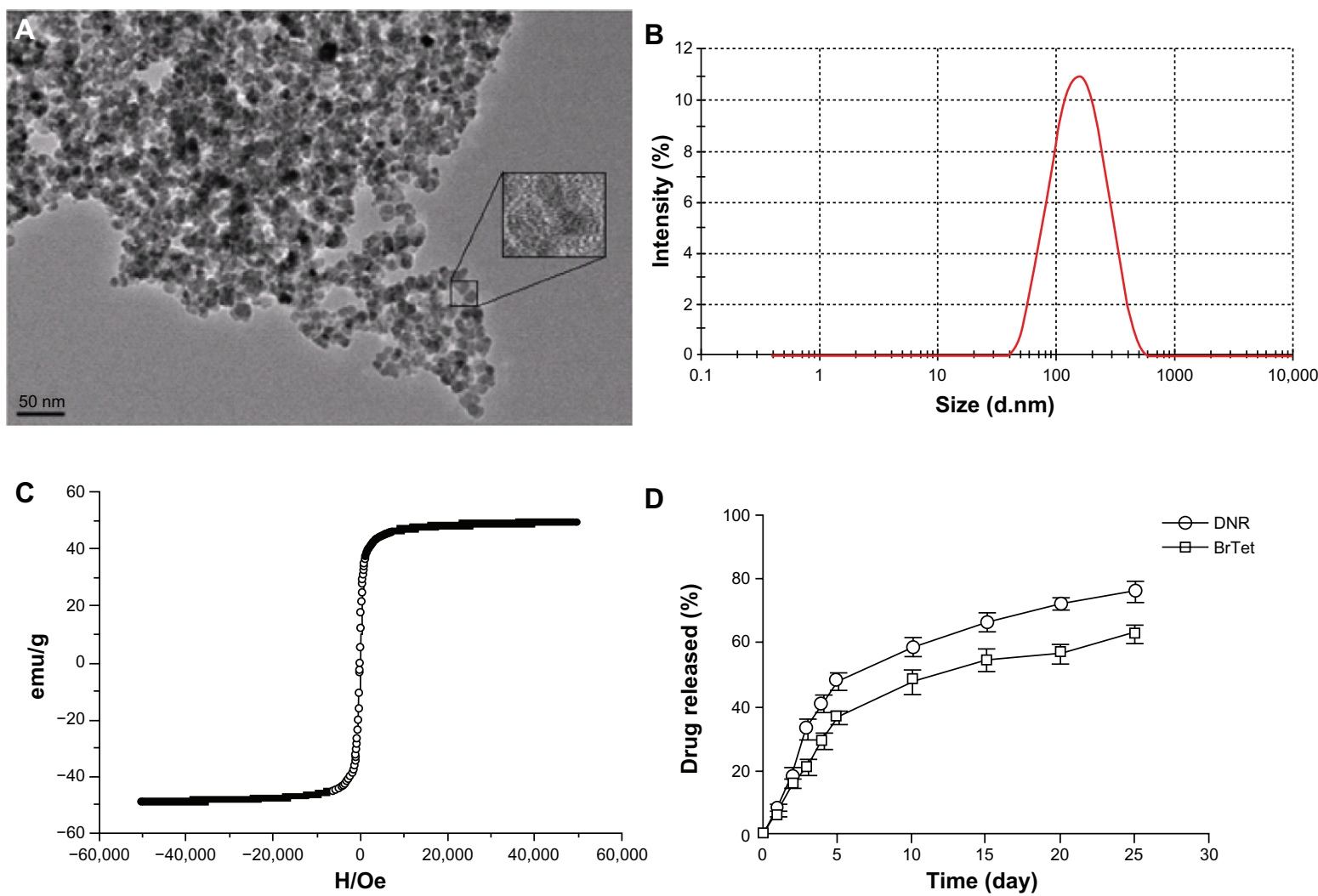

Figure I Physical characteristic of drug-loaded magnetic nanoparticles. (A) The nanoparticles under the transmission electron microscope. (B) Hydrodynamic particle size distribution of DNR/BrTet-loaded MNPs by Malvern Zetasizer Nano ZS90 (Malvern Instruments, Worcestershire, UK). (C) Magnetizaion as a function of field drug-loaded iron oxide nanoparticles by a vibration sample magnetometer. (D) Release of DNR and BrTet from MNPs under in-vitro conditions.

Abbreviations: DNR, daunorubicin; BrTet, 5-bromotetrandrin; MNP, magnetic nanoparticle. 
magnetization $\left(M_{s}\right)$ of $51.31 \mathrm{emu} / \mathrm{g}$ magnetic and coercivity $\left(H_{c}\right)$ of 15.67 Oe by VSM (Figure 1C).

\section{Drug loading and release}

Drug-loading capacity and encapsulation efficiency are shown in Table 1. The released behavior of DNR/BrTet-MNPs in Tween-PBS solution at $37^{\circ} \mathrm{C}$ was shown in Figure 1D. The common burst release of DNR and BrTet were both observed in the first 5 days, of which about $48 \%$ was for DNR and $37 \%$ for BrTet. Interestingly, the drug-loaded MNPs were able to release drugs sustainably until 25 days.

\section{In vitro cytotoxicity on $\mathrm{K} 562 / \mathrm{A} 02$ cells of drug-loaded MNPs}

When incubated with MNPs (less than $100 \mu \mathrm{g} / \mathrm{mL}$ ) or BrTet (less than $2 \mu \mathrm{g} / \mathrm{mL}$ ) for 48 hours, obvious cytotoxic effects on K562/A02 cells were not observed (Table 2). Both DNR alone and in combination with MNPs significantly inhibited the growth of $\mathrm{K} 562 / \mathrm{A} 02$ cells, and the $\mathrm{IC}_{50}$ values were $19.11 \pm 1.05 \mu \mathrm{g} / \mathrm{mL}$ and $10.71 \pm 0.62 \mu \mathrm{g} / \mathrm{mL}$, respectively. Notably, when DNR was co-administrated with BrTet (DNR: BrTet $=1: 1 \mathrm{w} / \mathrm{w}$ ), the cytotoxicity to K562/ A02 cells was significantly enhanced and the $\mathrm{IC}_{50}$ value was $1.01 \pm 0.17 \mu \mathrm{g} / \mathrm{mL}$. DNR/BrTet-co-loaded MNPs also displayed a dose-dependent antiproliferative activity, and the $\mathrm{IC}_{50}$ value was $8.40 \pm 1.26 \mu \mathrm{g} / \mathrm{mL}$ (Figure 2).

\section{Fluorescent microscope for cellular uptake of drug}

After incubation for 48 hours, high red fluoresce from DNR was seen localized in the cytoplasm of cells treated with DNR alone or DNR-MNPs, indicating that cells endocytosed and/or pinocytosed free DNR or DNR-MNPs (Figure 3A and B). When being combined with BrTet, a prominent increased red fluorescence was observed in the nucleus (Figure 3C). The nuclear and cytoplasm localization of DNR was observed in cells treated with DNR/BrTet-MNPs (Figure 3D).

Table I Drug loading of DNR and BrTet in MNPs $(n=3$, mean \pm standard deviation)

\begin{tabular}{|c|c|c|c|c|}
\hline \multicolumn{2}{|c|}{ DNR (\% w/w) } & \multicolumn{2}{|c|}{ BrTet (\% w/w) } & \multirow{2}{*}{$\begin{array}{l}\text { Total drug } \\
\text { loading }(\% \mathrm{w} / \mathrm{w})\end{array}$} \\
\hline Added & Loaded & Added & Loaded & \\
\hline 0.0 & 0.0 & 10.0 & $6.15 \pm 0.65$ & $6.15 \pm 0.65$ \\
\hline 5.0 & $4.60 \pm 0.39$ & 5.0 & $3.65 \pm 0.80$ & $8.25 \pm 1.13$ \\
\hline 10.0 & $7.62 \pm 0.47$ & 0.0 & 0.0 & $7.62 \pm 0.47$ \\
\hline
\end{tabular}

Abbreviations: DNR, daunorubicin; BrTet, 5-bromotetrandrin; MNP, magnetic nanoparticle.
Table 2 Effect of MNPs and BrTet on K562/A02 cells for 48 hours ( $n=3$, mean \pm standard deviation)

\begin{tabular}{|c|c|c|c|}
\hline \multicolumn{2}{|l|}{ MNPs } & \multicolumn{2}{|l|}{ BrTet } \\
\hline $\begin{array}{l}\text { Concentration } \\
(\mu \mathrm{g} / \mathrm{mL})\end{array}$ & $\begin{array}{l}\text { Relative } \\
\text { growth } \\
\text { rate (\%) }\end{array}$ & $\begin{array}{l}\text { Concentration } \\
(\mu \mathrm{g} / \mathrm{mL})\end{array}$ & $\begin{array}{l}\text { Relative } \\
\text { growth } \\
\text { rate (\%) }\end{array}$ \\
\hline 6.25 & $97.12 \pm 1.31$ & 0.1 & $103.46 \pm 2.09$ \\
\hline 12.5 & $89.06 \pm 0.36$ & 0.5 & $105.02 \pm 2.78$ \\
\hline 25 & $84.45 \pm 1.24$ & 1 & $102.95 \pm 2.54$ \\
\hline 50 & $85.87 \pm 4.50$ & 2 & $91.87 \pm 1.98^{a}$ \\
\hline 100 & $81.79 \pm 1.91^{b}$ & 5 & $14.02 \pm 2.04$ \\
\hline
\end{tabular}

Notes: aRelative growth rate was more than $90 \%$ when BrTet was less than $2 \mu g / \mathrm{mL}$. 'Relative growth rate was more than $80 \%$ when MNPs alone was less than $100 \mu \mathrm{g} / \mathrm{mL}$. Abbreviations: DNR, daunorubicin; BrTet, 5-bromotetrandrin; MNPs, magnetic nanoparticles.

\section{Function of $\mathrm{P}$-gP in K562/A02 cells}

R123 uptake was increased in K562/A02 cells treated with either BrTet alone or DNR/BrTet compared with DNR alone, and the increase was also observed in cells treated with either BrTet-MNPs or DNR/BrTet-MNPs compared with DNR-MNPs $(P<0.05)$. In addition, there were significant differences among BrTet, DNR/BrTet, BrTet-MNPs, and DNR/BrTet-MNPs $(P<0.05)$ (Figure 5).

\section{Expression of mdrl/P-gp in K562/A02 cells}

Compared with control group, the transcriptions of mdr1 mRNA were significantly downregulated by nearly $26 \%$ in the combination of DNR with BrTet (DNR/BrTet 1) group and by $13 \%$ in the DNR/BrTet-MNPs group $(P<0.05)$ (Figure 5A). The levels of P-gp were significantly downregulated by nearly $42 \%$ in the DNR/BrTet group and by $32 \%$ in DNR/BrTet-MNPs group $(P<0.05)$ (Figure 4B).

\section{Discussion}

Currently, systemic chemotherapy is still the main treatment option for hematologic malignancies, but the disturbing fact is that drug-resistant cells usually lead to therapeutic failure. MDR is a multifactorial problem involving several genes acting either alone or in concert with other factors. For example, the increased expression of MDR1 drug transporter gene coding for P-gp pump, MDR-related protein, and topoisomerase II has been associated with the MDR phenotype. ${ }^{24} \mathrm{~A}$ lot of chemotherapeutic agents such as anthracyclines or paclitaxel have been proved to be capable of inducing drug resistance via overexpression mdr1/P-gp pathway ${ }^{25}$ which is one of the major mechanisms of MDR. Although several MDR reversal agents have been applied in laboratory studies and clinical trials, there is still no significant improvement. Notably, 

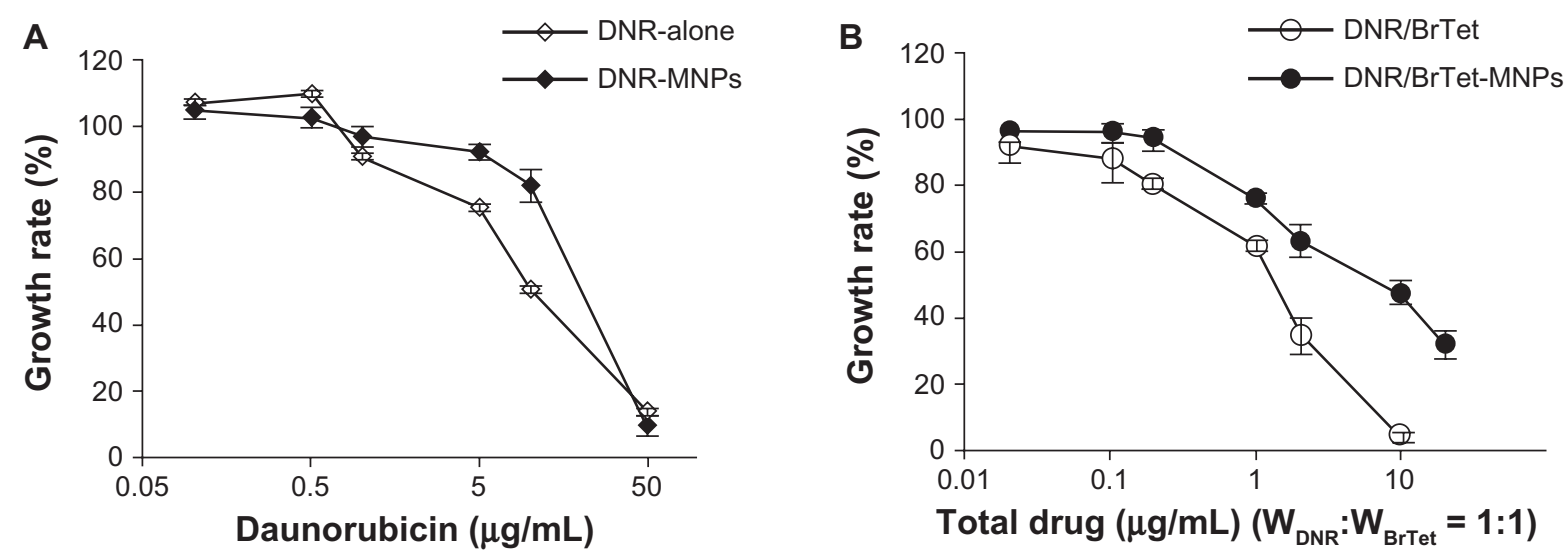

Figure 2 Cytotoxicity effects on K562/A02 cells for 48 hours. (A) DNR with or without MNPs. (B) Co-administration of DNR and BrTet with or without MNPs. Abbreviations: DNR, daunorubicin; BrTet, 5-bromotetrandrin; MNP, magnetic nanoparticle; W, weight.

BrTet, an excellent reversal of P-gp mediated MDR, ${ }^{23,26}$ significantly enhanced the anticancer activity of doxorubicin in vitro by inhibiting the overexpression of P-gp. ${ }^{27}$

To overcome drug resistance and reduce the side effects during chemotherapy, nanotechnology holds promising potential, utilizing targeted drug delivery. In this study, the ability of a novel chemotherapy formulation (DNR/BrTet-
MNPs) to overcome P-gp-mediated drug resistance in K562/ A02 cells was investigated, and the efficacy evaluated. First, an anticancer drug and a MDR reversal agent were encapsulated in the pluronic-OA-iron oxide nanoparticles, which are roughly spherical from $135 \mathrm{~nm}$ and have superparamagnetic with $M_{s}$ of $51.31 \mathrm{emu} / \mathrm{g}$ magnetic and $H_{c}$ of $15.67 \mathrm{Oe}$. In this formulation, DNR and BrTet are stored in the OA shell

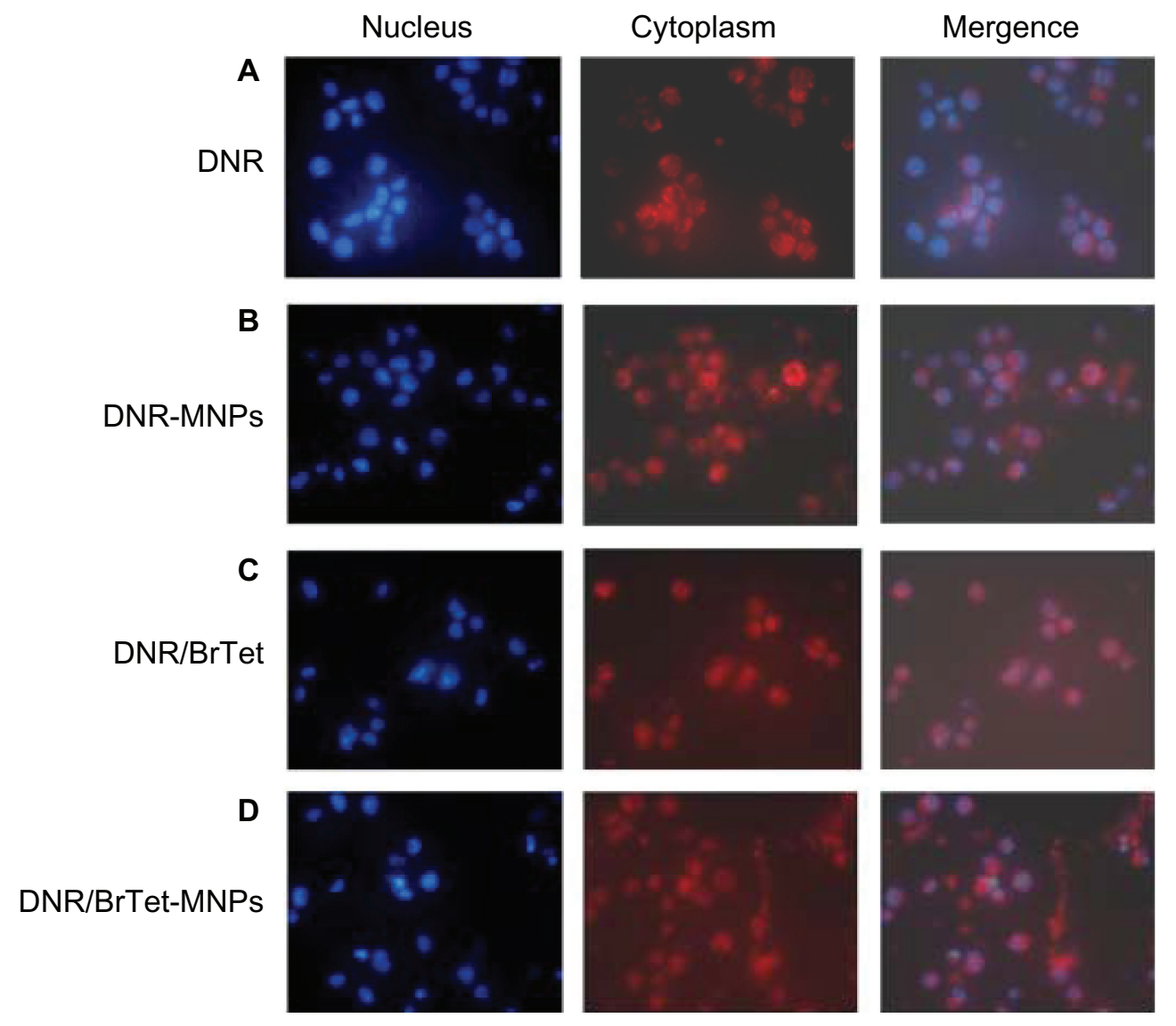

Figure 3 Subcellular distribution of DNR in K562/A02 cells treated with DNR alone, DNR-MNPs, DNR/BrTet, and DNR/BrTet-MNPs under fluorescence microscope. Abbreviations: DNR, daunorubicin; BrTet, 5-bromotetrandrin; MNP, magnetic nanoparticle. 
A
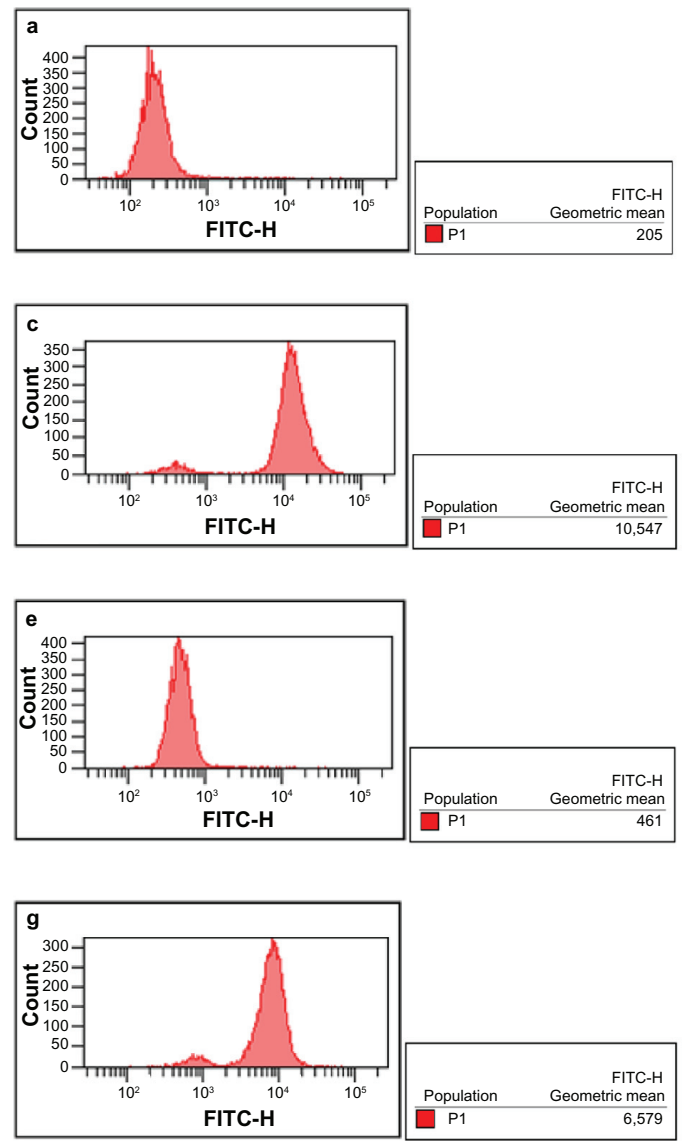
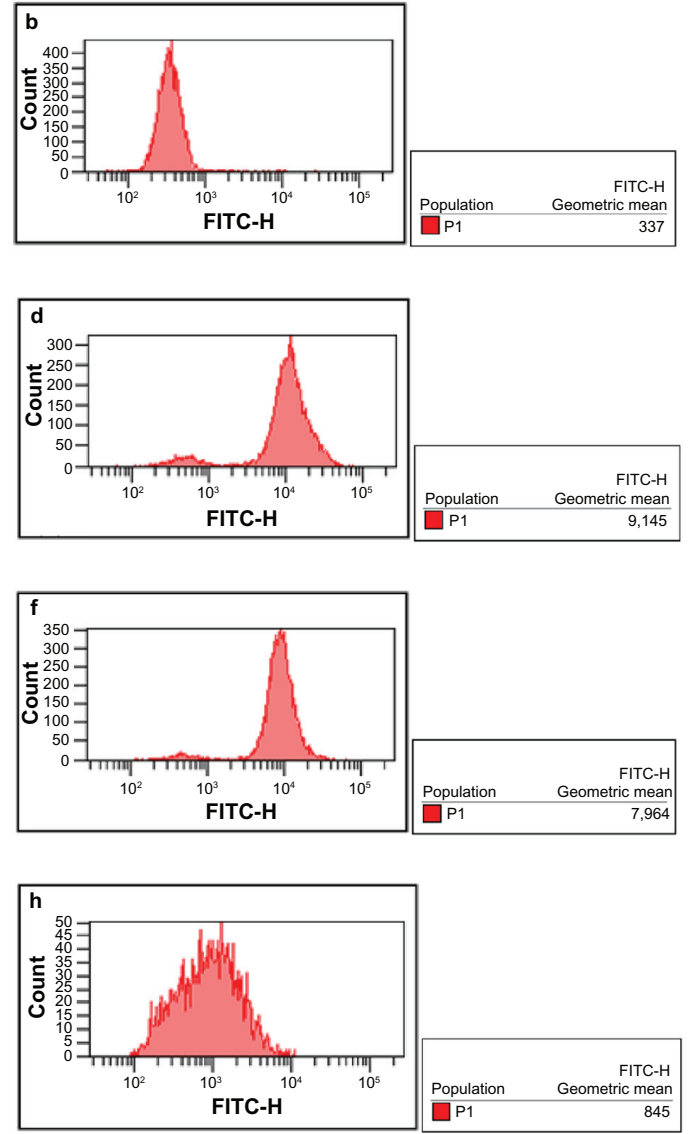

B

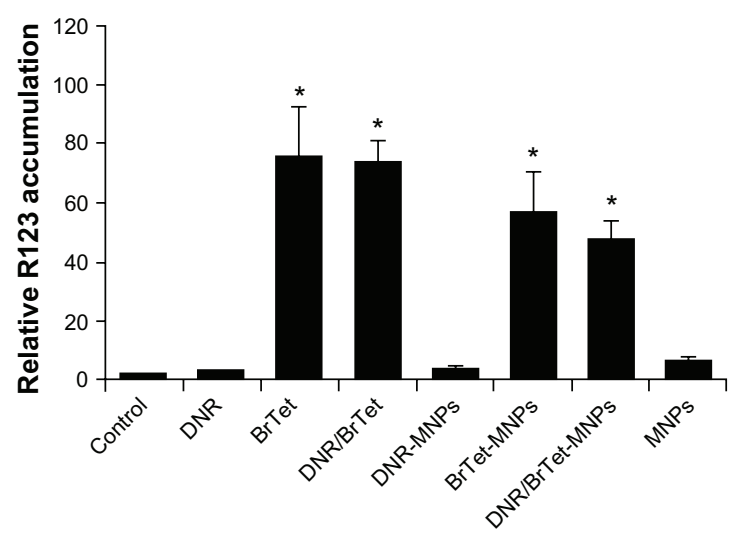

Figure 4 Function of P-gP in K562/A02 cells. (A) Intracellular fluorescence intensity (FI) associated with RI23 in treated cells: (a) control; (b) DNR; (c) BrTet; (d) DNR/ BrTet; (e) DNR-MNPs; (f) BrTet-MNPs; (g) DNR/BrTet-MNPs; (h) MNPs. (B) Relative RI23 accumulation in K562/A02 cells. Data are presented as means \pm standard deviation $(\mathrm{n}=3)$ and analyzed using one-way analysis of variance.

Note: $* p<0.05$, when compared with control group.

Abbreviations: BrTet, 5-bromotetrandrin; DNR, daunorubicin; FITC-H, heavy chain fluorescein isothiocyanate; MNP, magnetic nanoparticle; P-gp, P-glycoprotein; RI23, rhodamine I23; RT-PCR, reverse transcriptase polymerase chain reaction.

and released sustainably, where $\mathrm{OA}$ acts as a drug depot and Pluronic F-127, an amphiphilic synthetic polymer, is used to improve aqueous dispersity of the formulation, which makes the nanoparticles disperse well in water and prolongs the systemic circulation time and increases eventual localization efficiency of the drug-delivery system to the tumor site. ${ }^{28}$ In addition, it also acts as a drug depot system for the sustained release of drug and ensures effective dose in targeted tissue and reduces damage to other organs. Compared with coadministration of a P-gp inhibitor and an anticancer drug, ${ }^{29}$ in this present study the adoption of nanoparticles in the drug-delivery system made it possible for two drugs with different pharmacokinetics and tissue disposition to play roles at the same time and same place. 
A
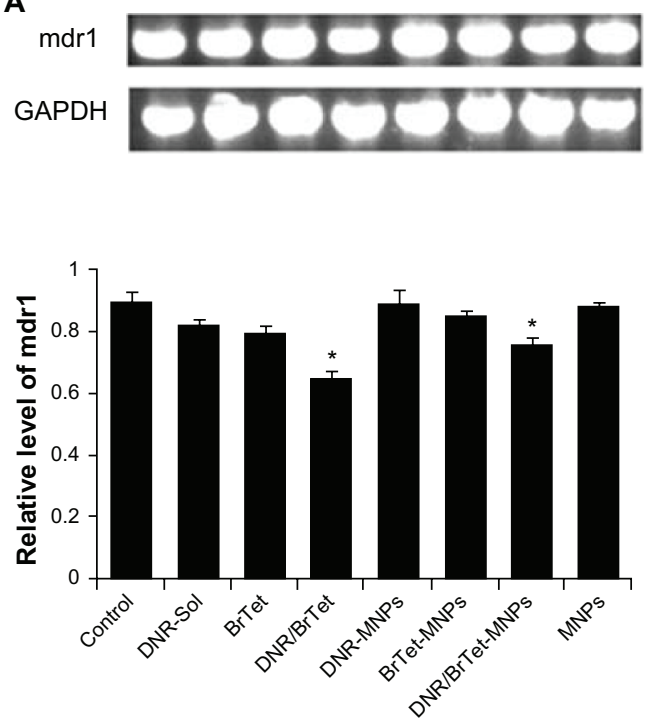

B
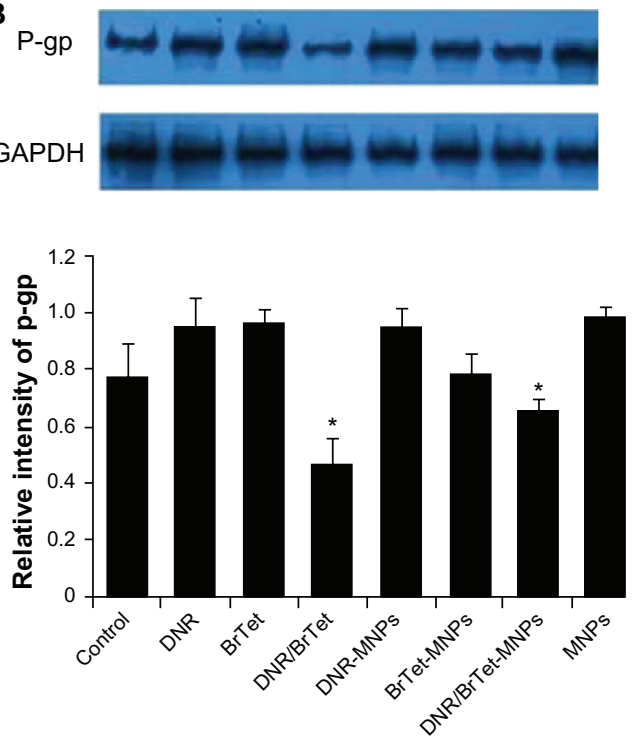

Figure 5 Transcription of mdrl and expressions of P-gp in K562/A02 cells. (A) Transcription of mdrl mRNA by RT-PCR; (B) expression of P-gp by western blotting assay. Note: $* P<0.05$, when compared with control group.

Abbreviations: BrTet, 5-bromotetrandrin; DNR, daunorubicin; GAPDH, glyceraldehyde 3-phosphate dehydrogenase; MNP, magnetic nanoparticle; P-gP, P-glycoprotein; RT-PCR, reverse transcriptase polymerase chain reaction.

Next, the effects of the formulation of a nanoparticle delivery system for overcoming drug-resistant K562/A02 in vitro was tested. In the present study, only about $19 \%$ of DNR and $16 \%$ of BrTet are released from MNPs in the first 2 days. The efficacy of DNR/BrTet-MNPs on K562/A02 cells at 48 hours was lower than that of DNR-BrTet, which may be down to the sustained release of drugs. Meanwhile, it is found that DNR/BrTet-MNPs show significant antiproliferative activity compared with DNR-MNPs, which is due to the reserve effect of BrTet. It is reasonable to see that red fluorescence intensity was primarily accumulated in the cytoplasm of K562/A02 cells treated with either DNR or DNR-MNPs, once DNR was co-administered with BrTet; red fluorescence intensity was increased significantly in the nucleus of K562/ A02 cells, indicating BrTet makes the increase of intracellular DNR accumulation through inhibiting the drug efflux pumps on the cell membrane, such as P-gp, and then DNR further diffuses into the nucleus. Interestingly, red fluorescent light both in the cytoplasm and nucleus was observed in K562/ A02 cells treated with DNR/BrTet-MNPs, suggesting that DNR and BrTet are released slowly from the nanoparticles in the cytoplasm after being taken up by cells, and then DNR slowly diffuses into the nucleus. Thus, the authors considered that DNR/BrTet-MNPs act as an intracellular depot system and promote sustained drug retention.

P-gp has been demonstrated to significantly reduce the toxicity of anticancer drugs by influencing their pharmacokinetics and pharmacodynamics. ${ }^{13}$ The present study clearly revealed that the transcription of $\mathrm{mdr} 1$ and expression of $\mathrm{P}$-gp were both downregulated in K562/A02 cells after incubation with either DNR/BrTet-MNPs or DNR/BrTet for 48 hours. The results of R123 uptake suggested that BrTet obviously inhibited the efflux of R123 by P-gp both in solution and in MNPs. Moreover, the accumulation of R123 in both BrTet and BrTet-MNPs groups was found to be lower than that in both DNR/BrTet-MNPs and DNR/BrTet. The possible explanation is that the decrease of R123 accumulation means cells treated with DNR/BrTet-MNPs or DNR/BrTet are undergoing apoptosis, for R123 is a fluorescent probe for monitoring the membrane potential of mitochondria. ${ }^{30}$ In summary, these results indicate that a combination of DNR and BrTet with or without MNPs prevent the development of mdr1 phenotype in K562/A02 cells in vitro. But the effect has not been observed by DNR in nanoparticles, which is to some extent controversial to that reported by Jain et al; in their study, doxorubicin loaded with nanoparticles showed antitumor efficacy on tumor cells. ${ }^{30,31}$ These opposing results suggest that there may be fundamental differences between cell types and the characteristic of sustained release of the formulation used in this study. Importantly, this novel magnetic iron oxide nanoparticle formulation co-loading DNR and BrTet with the excellent property of sustained release is superior to the prior polymerized MNPs with DNR and BrTet. ${ }^{23}$ All of these findings suggest the novel magnetic iron oxide nanoparticle formulation co-loading DNR and BrTet may be a successful platform to overcome MDR. 


\section{Conclusion}

The remarkable effect of the novel DNR/BrTet-MNPs formulation, acting as a drug depot system for the sustained release of the loaded DNR and BrTet on multidrug resistance leukemia K562/A02 cells would be a promising strategy for overcoming MDR.

\section{Acknowledgments}

This work was financially supported by National Key Basic Research Program 973 of China (No 2010CB732404) and National Nature Science Foundation of People's Republic of China (No 30740062 and No 30872970).

\section{Disclosure}

The authors report no conflicts of interest in this work.

\section{References}

1. Szakács G, Paterson JK, Ludwig JA, Booth-Genthe C, Gottesman MM. Targeting multidrug resistance in cancer. Nat Rev Drug Discov. 2006;5: 219-234.

2. Gottesman MM, Fojo T, Bates SE. Multidrug resistance in cancer: role of ATP dependent transporters. Nat Rev Cancer. 2002;2:48-58.

3. Loo TW, Clarke DM. Recent progress in understanding the mechanism of P-glycoprotein-mediated drug efflux. J Membr Biol. 2005; 206:173-185.

4. Fox E, Bates SE. Tariquidar (XR9576): a P-glycoprotein drug efflux pump inhibitor. Expert Rev Anticancer Ther. 2007;7:447-459.

5. Klopman G, Shi LM, Ramu A. Quantitative structure activity relationship of multidrug resistance reversal agents. Mol Pharmacol. 1997;52:323-334.

6. Sikic BI, Fisher GA, Lum BL, Halsey J, Beketic-Oreskovic L, Chen G. Modulation and prevention of multidrug resistance by inhibitors of P-glycoprotein. Cancer Chemother Pharmacol. 1997;40:S13-S19.

7. Kolitz JE, George SL, Marcucci G, et al. P-glycoprotein inhibition using valspodar (PSC-833) does not improve outcomes for patients younger than age 60 years with newly diagnosed acute myeloid leukemia: Cancer and Leukemia Group B study 19808. Blood. 2010;116:1413-1421.

8. Abraham J, Edgerly M, Wilson R, et al. A phase I study of the P-glycoprotein antagonist tariquidar in combination with vinorelbine. Clin Cancer Res. 2009;15:3574-3582.

9. Ruff P, Vorobiof DA, Jordaan JP, et al. A randomized, placebo-controlled, double-blind Phase 2 study of docetaxel compared to docetaxel plus zosuquidar (LY335979) in women with metastatic or locally recurrent breast cancer who have received one prior chemotherapy regimen. Cancer Chemother Pharmacol. 2009;64:763-768.

10. Dai CL, Xiong HY, Tang LF, et al. Tetrandrine achieved plasma concentrations capable of reversing MDR in vitro and had no apparent effect on doxorubicin pharmacokinetics in mice. Cancer Chemother Pharmacol. 2007;60:741-750.

11. Wang G, Lemos JR. Tetrandrine: a new ligand to block voltage-dependent Ca-2+ and Ca-2+-activated K+ channels. Life Sci. 1995;56:295-306.

International Journal of Nanomedicine

\section{Publish your work in this journal}

The International Journal of Nanomedicine is an international, peerreviewed journal focusing on the application of nanotechnology in diagnostics, therapeutics, and drug delivery systems throughout the biomedical field. This journal is indexed on PubMed Central, MedLine, CAS, SciSearch $₫$, Current Contents ${ }^{\circledR} /$ Clinical Medicine,
12. Fu L, Liang Y, Deng L, et al. Characterization of tetrandrine, a potent inhibitor of P-glycoprotein-mediated multidrug resistance. Cancer Chemother Pharmacol. 2004;53:349-356.

13. Jin J, Wang FP, Wei H, Liu GT. Reversal of multidrug resistance of cancer through inhibition of P-glycoprotein by 5-bromotetrandrine. Cancer Chemother Pharmacol. 2005;55:179-188.

14. Hu YL, Fu YH, Tabata Y, Gao JQ. Mesenchymal stem cells: a promising targeted-delivery vehicle in cancer gene therapy. J Control Release. 2010;147:154-162.

15. Kim KY. Nanotechnology platforms and physiological challenges for cancer therapeutics. Nanomedicine. 2007;3:103-110.

16. Sahoo SK, Labhasetwar V. Nanotech approaches to drug delivery and imaging. Drug Discov Today. 2003;8:1112-1120.

17. Mahmoudi M, Simchi A, Imani M, Milani AS, Stroeve P. Optimal design and characterization of superparamagnetic iron oxide nanoparticles coated with polyvinyl alcohol for targeted delivery and imaging. J Phys Chem B. 2008;112:14470-14481.

18. Endicott JA, Ling V. The biochemistry of P-glycoprotein-mediated multidrug resistance. Annu Rev Biochem. 1989;58:137-171.

19. Lothstein L, Israel M, Sweatman TW. Anthracycline drug targeting: cytoplasmic versus nuclear - a fork in the road. Drug Resist Updat. 2001;4:169-177.

20. Guo J, Zhou J, Ying X, et al. Effects of stealth liposomal daunorubicin plus tamoxifen on the breast cancer and cancer stem cells. J Pharm Pharmaceut Sci. 2010;13:136-151.

21. Wang J, Chen BA, Cheng J, et al. Synthesis and antitumor efficacy of daunorubicin-loaded magnetic nanoparticles in vitro. Int $J$ Nanomedicine. 2010;6:203-211.

22. Xu JP, Ji J, Chen WD, Shen JC. Novel biomimetic polymersomes as polymer therapeutics for drug delivery. J Control Release. 2005;107: 502-512.

23. Cheng J, Wu WW, Chen BA, et al. Effect of magnetic nanoparticles of $\mathrm{Fe}_{3} \mathrm{O}_{4}$ and 5-bromotetrandrine on reversal of multidrug resistance in K562/A02 leukemic cells. Int J Nanomedicine. 2009;4:209-216.

24. Ling V. Multidrug resistance: molecular mechanisms and clinical relevance. Cancer Chemother Pharmacol. 1997;40:S3-S8.

25. Horwitz SB, Cohen D, Rao S, Ringel I, Shen H J, Yang C P. Taxol: mechanisms of action and resistance. J Natl Cancer Inst Monogr. 1993;15: 55-61.

26. Chen BA, Cheng J, Wu YN, et al. Reversal of multidrug resistance by magnetic $\mathrm{Fe}_{3} \mathrm{O}_{4}$ nanoparticle copolymerizating daunorubicin and 5-bromotetrandrine in xenograft nude-mice. Int $J$ Nanomedicine. 2009; 4:73-78.

27. Chen BA, Wang J, Ding JH, et al. Reversal of P-glycoprotein-dependent resistance to adriamycin by 5-bromotetrandrine in K562/A02 cell line [abstract]. J Clin Oncol. 2008;26(Suppl):13557.

28. Iyer AK, Khaled G, Fang J, Maeda H. Exploiting the enhanced permeability and retention effect for tumor targeting. Drug Discov Today. 2006;11:812-818.

29. Miettinen S, Grenman S, Ylikomi T. Inhibition of P-glycoproteinmediated docetaxel efflux sensitizes ovarian cancer cells to concomitant docetaxel and SN-38 exposure. Anticancer Drugs. 2009;20:267-276.

30. Susa M, Iyer AK, Ryu K, et al. Doxorubicin loaded polymeric nanoparticulate delivery system to overcome drug resistance in osteosarcoma. BMC Cancer. 2009;9:399.

31. Jain TK, Morales MA, Sahoo SK, et al. Iron oxide nanoparticles for sustained delivery of anticancer agents. Mol Pharm. 2005;2:194-205.

\section{Dovepress}

Journal Citation Reports/Science Edition, EMBase, Scopus and the Elsevier Bibliographic databases. The manuscript management system is completely online and includes a very quick and fair peer-review system, which is all easy to use. Visit http://www.dovepress.com/ testimonials.php to read real quotes from published authors. 